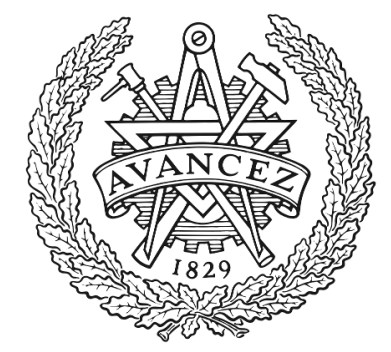

\title{
CHALMERS
}

UNIVERSITY OF TECHNOLOGY

\section{The influence of tyre lateral force for control allocation of yaw torque}

Downloaded from: https://research.chalmers.se, 2023-04-26 10:13 UTC

Citation for the original published paper (version of record):

Yang, D., Jonasson, M. (2020). The influence of tyre lateral force for control allocation of yaw torque. Lecture Notes in Mechanical Engineering: 1536-1542.

http://dx.doi.org/10.1007/978-3-030-38077-9_176

N.B. When citing this work, cite the original published paper. 


\title{
The Influence of Tyre Lateral Force for Control Allocation of Yaw Torque
}

\author{
Derong Yang ${ }^{1}$ and Mats Jonasson ${ }^{2}$ \\ 1 Volvo Car Group, Department of Active Safety and Vehicle Dynamics, \\ Gothenburg, 405 31, Sweden \\ derong. yang@volvocars.com, \\ 2 Department of Mechanics and Maritime Sciences, Chalmers University of \\ Technology, 41296 Gothenburg, Sweden
}

\begin{abstract}
The present paper provides a thorough analysis and reveals the yaw torque generated by tyre lateral forces, due to the well-known combined slip effect. The indirect yaw torque here is captured by a tyre model simplification designed for the real-time control allocation purpose. Experiments were carried out by a driving robot, controlling steering wheel, gas and brake pedal at various manoeuvres. The test vehicle is equipped with high precision measurement-wheel mounted at each wheel. It was found that the simplified model correlates with the experimental results, where the relation between the wheel torque distribution of front/rear axles and the yaw torque generated by tyre lateral forces are highly dependent on the vehicle lateral acceleration and drive torque request.
\end{abstract}

Keywords: Optimal Yaw Torque Control, Tyre Model, Control Allocation, Indirect Yaw torque

\section{Introduction}

In the recent decades, owing to the development of electric vehicles and Torque Vectoring (TV) technologies, vehicle stability and yaw response can be simultaneously optimized based on the standard direct yaw torque control algorithm for example Torque Vectoring as in [1-4], without comprising other crucial vehicle properties such as fuel economy and ride comfort. The cascading of the desired yaw torque to the individual wheel torque is commonly included in the framework of control allocation. Due to the complexity of the tyre forces at dynamic driving, the control allocation algorithms nowadays on board do not consider the combined-slip effect of the tyre. Previous research has tackled this problem in various ways $[5,6]$, however the allocation models including tyre behaviours used in the controllers were not explicitly tested and validated. The present paper provides a detailed experimental quantification and qualification of how tyre forces can influence the yaw torque balance for Torque Vectoring alike functions. 


\section{Control allocation architecture}

A number of functions in a premium passenger car handles the wheel torque distribution among front/rear axle and left/right side. In order to centralize the wheel torque distribution and also include the effect of combined slip, a simplified theory was proposed and presented by the authors in [7]. The control architecture as a whole, can be seen in Fig. 2. The centralized algorithm solves the allocation problem

$$
\begin{array}{ll}
\underset{u}{\operatorname{minimize}} & (B u-v)^{T} Q(B u-v)+u^{T} R u \\
\text { subject to } & C u \leq b
\end{array}
$$

where the unknown $u$ consists of torques from the vehicle's actuators, such as an internal combustion engine, electric machines and friction brakes, in order to meet the desired vehicle longitudinal traction force as well as yaw torque $v=\left[T_{x}, M_{z}\right]^{\mathrm{T}}$ subjected to the attainable wheel torques $b$. The generation of yaw torque is defined as

$$
M_{z}=M_{z}^{\mathrm{dir}}+M_{z}^{\mathrm{ind}}
$$

where the direct yaw torque, $M_{z}^{\text {dir }}$, is generated by the longitudinal tyre forces and the indirect yaw torque, $M_{z}^{\text {ind }}$ is generated by the lateral tyre forces as a combined slip effect of the longitudinal ones. In [7] it was further assumed that the indirect yaw torque around an operational point of constant lateral acceleration and traction torque could be approximated to

$$
M_{z}^{\text {ind }}=k(\epsilon-a), \quad \epsilon=\frac{T_{x, \text { rear }}}{T_{x, \text { rear }}+T_{x, \text { front }}}
$$

where $k$ and $a$ are tyre dependent parameters strongly influenced by the tyre combined slip mechanism. The Eq. 3 should be interpreted such that the indirect yaw torque could be influenced by changing the front to rear distribution of tractive force without changing either the total tractive force nor the lateral acceleration. One implication of Eq. 3 is that a re-distribution of tractive force from the front to the rear axle will increase yaw torque and in turn the understeering of the vehice will be reduced.

The indirect yaw torque is expressed as

$$
M_{z}^{\text {ind }}=\left(f_{y}^{F L}+f_{y}^{F R}\right) \cos \left(\delta_{f}\right) l_{f}+\left(f_{y}^{F L}-f_{y}^{F R}\right) \sin \left(\delta_{f}\right) w / 2-\left(f_{y}^{R L}+f_{y}^{R R}\right) l_{r}
$$

where the super-scripts $\{F L, F R, R L, R R\}$ indicates the front $(F)$, rear $(R)$, left $(\mathrm{L})$ and right $(\mathrm{R})$ tyre. The steering angle is denoted $\delta_{f}, l_{f}$ and $l_{r}$ are the distances from center of gravity to the front and rear axle respectively, $w$ is the track width.

In [7] the conceptual and simplified hypothesis, as illustrated in Fig. 1, was introduced to describe how indirect yaw torque is dependent on tractive axle force distribution.

The work in this paper has been done to verify if the hypothesis in Fig. 1 holds by experimental tyre force measurements. Of particular interest is if the 


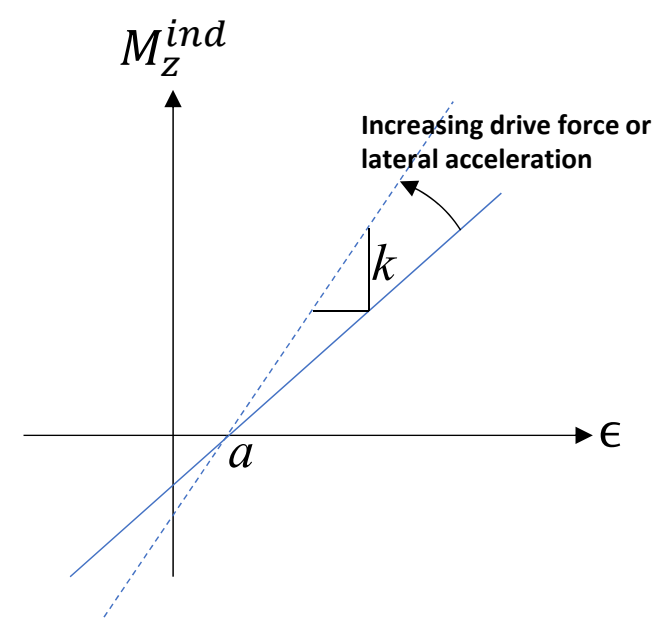

Fig. 1: The indirect yaw torque dependent on the tractive axle force distribution.

assumption done in Eq. 3 is good enough and to quantify when larger deviations are expected.

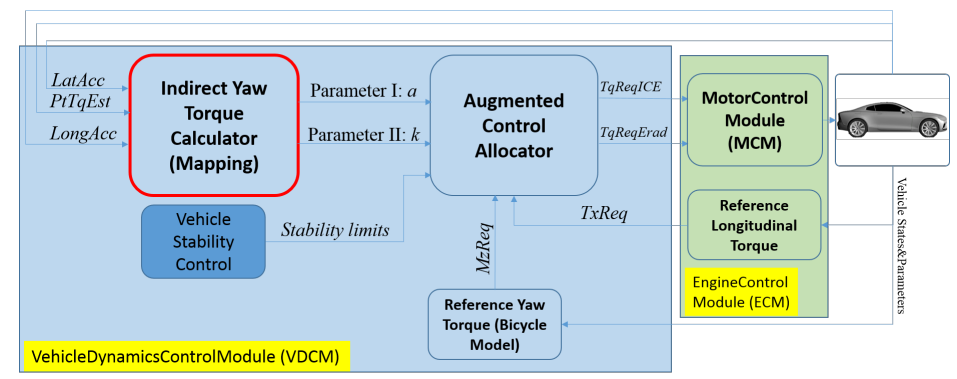

Fig. 2: Architecture of yaw torque control allocation. 


\section{Experiment}

\subsection{Test vehicle}

In order to measure the tyre forces and be able to repeat measurements with high precision, a test vehicle was equipped with torque and force sensing measuringwheels combined with a driving robot, see Fig. 3.
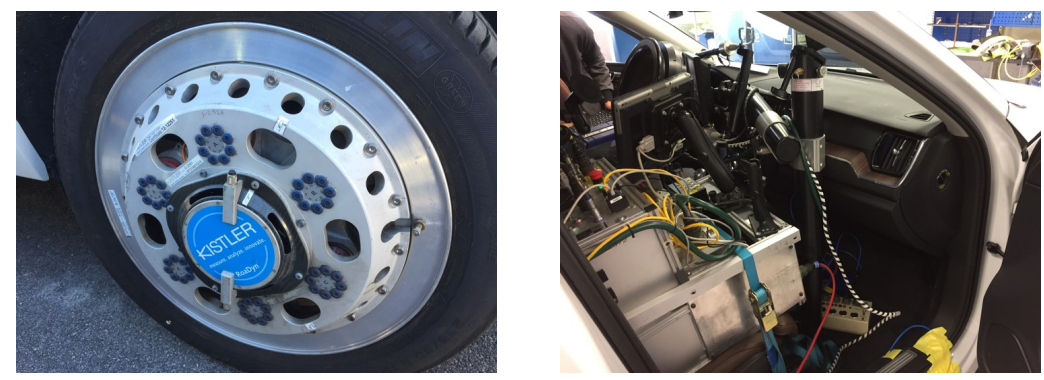

Fig. 3: Measurement wheels and driving robot used in the experiment.

The actual test vehicle is a Volvo XC60 T8 hybrid which has one ICE at the front axle and one electric motor at the rear axle. The electric motor has the top power and torque outputs approx. as $85 \mathrm{~kW}$ and $240 \mathrm{Nm}$, which corresponds to about $1800 \mathrm{Nm}$ at the wheel. The torques distribution between the front and rear axles could be calibrated by a development software in the vehicle's electronic control units.

\section{$3.2 \quad M_{z}^{\text {ind }}$ dependencies}

In Fig. 4, the indirect yaw torque is measured at a number of power-on-curve manoeuvres with different lateral accelerations and axle torque distribution configurations, where the throttle input is kept as the same i.e. $60 \%$. It shows that the higher the $a_{y}$, the larger the $M_{z}^{\text {ind }}$, given a similar total drive torque $T_{x}$. As noted by the text boxs in Fig. 4 , even if the throttle input is unchanged, $T_{x}$ could vary significantly at different axle torque distribution strategies. For instance, at $a_{y}=3\left[\mathrm{~m} / \mathrm{s}^{2}\right]$, FWD with ICE at the front axle, could deliver up to $5151 \mathrm{Nm}$, while RWD with electric motor stays only at $3667 \mathrm{Nm}$. This explains the lower $M_{z}^{\text {ind }}$ even with more drive torque allocated to the rear axle. Another observation is that for RWD case, at more aggressive lateral acceleration the indirect yaw torque becomes less, e.g at $a_{y}=5\left[\mathrm{~m} / \mathrm{s}^{2}\right]$ as compared to $a_{y}=4\left[\mathrm{~m} / \mathrm{s}^{2}\right]$. This reveals clearly a trade-off between longitudinal and lateral tyre forces due to combined slip effect, where higher lateral acceleration entering the curve limits the built-up of longitudinal forces, hence lower $M_{z}^{\text {ind }}$ can be generated. We see this as an indication of limited yaw influence by indirect yaw torque at AWD, 
where tyre lateral force can be easily saturated at high lateral acceleration and potential instability can be monitored.

Similarly, in Fig. 5, the indirect yaw torque is measured at a number of poweron-curve manoeuvres with different throttle inputs and axle torque distribution configurations, where the same lateral acceleration is reached i.e. $a_{y}=5\left[\mathrm{~m} / \mathrm{s}^{2}\right]$. It shows that the higher the throttle input, the larger the $M_{z}^{\text {ind }}$, given the same $a_{y}$. It is again noticed that the distribution of drive torque to the rear axle is highly limited by the power capacities of the electric motor at the axle, as well as the stability limits due to tyre slip.

The results of the closed-loop controller with the control allocation method described above, is shown by the AWD data points on the line connected by FWD and RWD cases. The hypothesis as presented in [7] was that indirect yaw torque can have a linear correlation with the longitudinal torque distribution, and its gradient is dependent on both the lateral acceleration and total drive torque. This would mean that any AWD case is lying on the line between FWD and RWD cases. Fig. 4 and Fig. 5 have given the evidence of this hypothesis, even though the actual drive torque was hardly maintained the same.

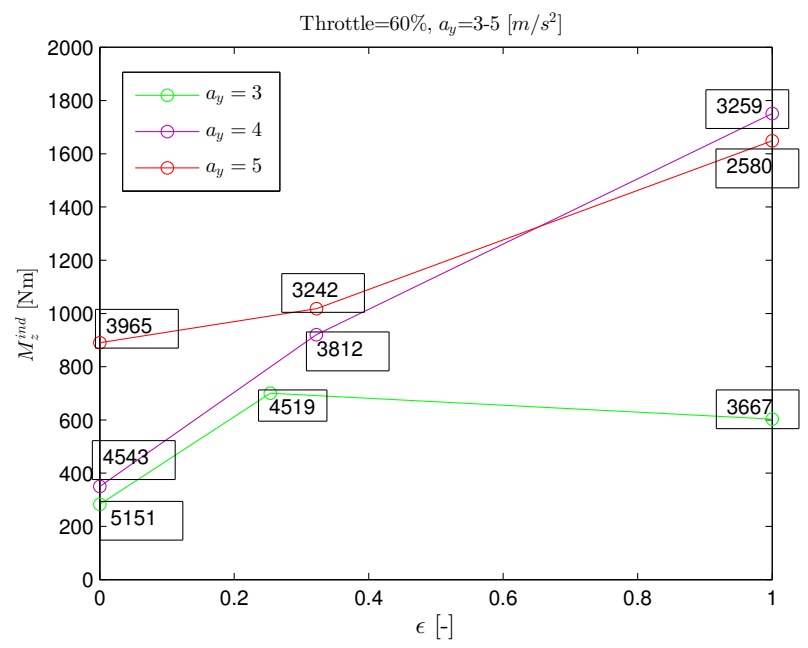

Fig. 4: Indirect yaw torque at different lat. accelerations and long. torque distribution. Text box denotes the total drive torque $T_{x}$.

\subsection{Exemplar scenarios results}

Fig. 6 below shows one example, where the ground truth of indirect yaw torque of three wheel torque distributions are shown. During the power-on-curve manoeuvre as shown by the vehicle states plot on the right, RWD $(\epsilon=1)$ here in 


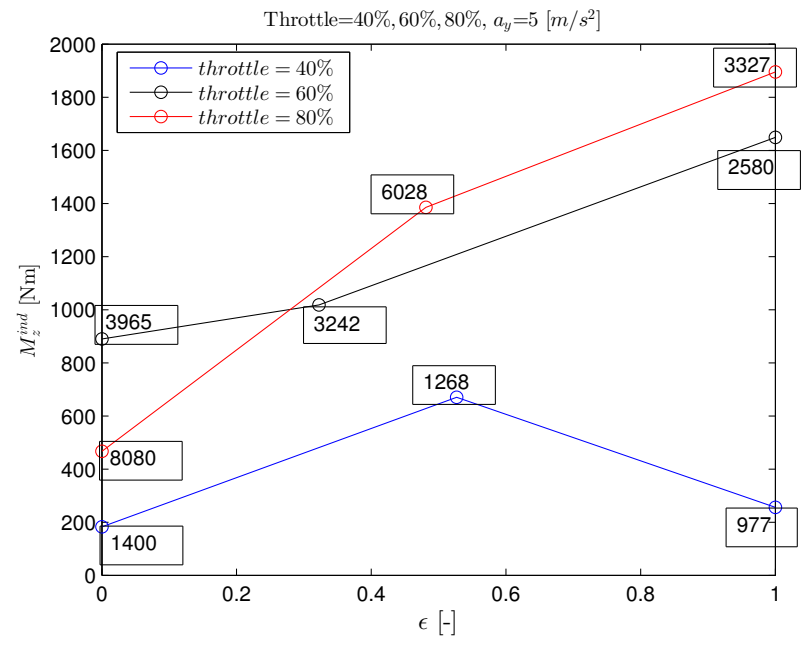

Fig. 5: Indirect yaw torque at different throttle inputs and long. torque distribution. Text box denotes the total drive torque $T_{x}$.

this case provides the highest indirect yaw torque, while $\operatorname{FWD}(\epsilon=0)$ provides the lowest amount; AWD case arrives in the middle as expected.
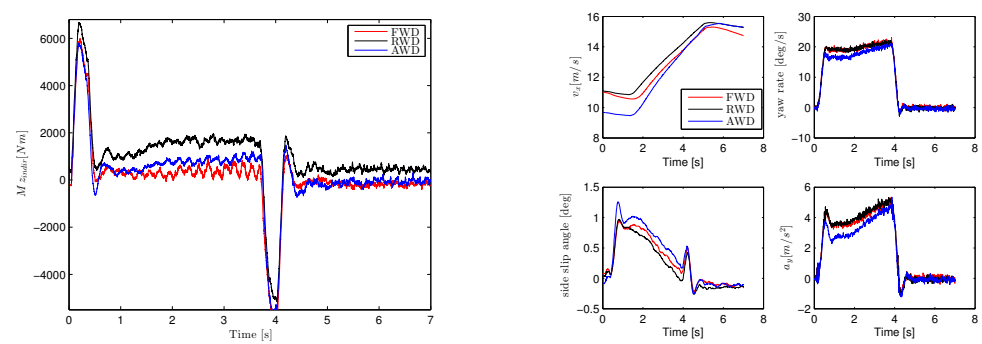

Fig. 6: Results driving with different axle torque distribution, at $60 \%$ and $a_{y}=4\left[\mathrm{~m} / \mathrm{s}^{2}\right]$.

Another example is shown below in Fig.7, at even higher drive torque request i.e. with $80 \%$ throttle $a_{y}=5\left[\mathrm{~m} / \mathrm{s}^{2}\right]$. It is interesting to note that at this nearlimit handling case, the FWD configuration generates negative yaw torque which makes the car fairly understeered. Furthermore, as shown in the vehicle speed diagram of Fig.7, even if RWD could not produce as much tractive force as compared to FWD and AWD, its influence of resultant tyre lateral force on yaw torque is prominent. Observe that with all driveline configurations, similar yaw response is seen in the yaw rate diagram, given different indirect yaw torque; this 
is due to the fact that certain direct yaw torque is generated by the differential slip controller.


Fig. 7: Results driving with different axle torque distribution, at $80 \%$ and $a_{y}=5\left[\mathrm{~m} / \mathrm{s}^{2}\right]$.

\section{Conclusions}

This work is an experimental study on how indirect yaw torque can be controlled by re-distributing tractive torque axle-wise. Different axle force distributions have been tested on a hybrid vehicle instrumented with force and torque measurement wheels and a driving robot. The investigation have had challenges controlling axle torque accurately which made the the verification more complicated. However, the experiments have confirmed that the indirect yaw torque increases approximately linearly when the axle torque distribution factor $\epsilon$ is increased. Further on, the indirect yaw torque is increased for an increased total tractive torque and lateral acceleration.

Future work is to further develop the indirect yaw torque calculator in the closed-loop controller, and perform experiments with measurement wheels to directly validate the accuracy and efficiency of the control allocation algorithm.

\section{References}

1. Gruber, P. et al.: Energy Efficient Torque Vectoring Control. Proc. of AVEC16, 2016, Munich, Germany.

2. Sun, P. et al.: Analysis of Camber Control and Torque Vectoring to Improve Vehicle Energy Efficiency. Proc. Of IAVSD17, 2017, Rockhampton, Australia.

3. Novellis, L. De. et al.: Direct Yaw torque Control Actuated through Electric Drivetrains and Friction Brakes: Theoretical Design and Experimental Assessment, Mechatronics, No. 26, 2015, pp. 115.

4. Sawase, K. et al.: Left-Right Torque Vectoring Technology as the Core of Super All Wheel Control (S-AWC). Mitsubishi Motors Technical Review, No. 18, 2006, pp. 1623. 
5. Gordon, T. et al.: Robust Implementation of Automated Collision Avoidance Using an Updating Particle Reference. Proc. of AVEC18, 2018, Beijing, China.

6. Frasch J. V. et al.: An Auto-generated Nonlinear MPC Algorithm for Real-Time Obstacle Avoidance of Ground Vehicles. Proc. 2013 European Control Conference (ECC), July 17-19, 2013, Zürich, Switzerland.

7. Yang, D. et al.: Torque Vectoring Control for Progressive Cornering Performance in AWD Electric Vehicles. Proc. of AVEC18, 2018, Beijing, China. 